\title{
Caracterização físico-química, nutricional e compostos bioativos de romã
}

\author{
Physical-chemical, nutritional characterization and bioactive compounds of pomegranate \\ Caracterización físico-química, nutricional y compuestos bioactivos de la granada
}

Recebido: 03/11/2021 | Revisado: 11/11/2021 | Aceito: 20/01/2022 | Publicado: 22/01/2022

\author{
Juliana Aparecida dos Santos \\ ORCID: https://orcid.org/0000-0001-5086-1536 \\ Universidade Estadual Paulista, Brasil \\ E-mail: juliana.aparecida@unesp.br \\ Andres Felipe Gaona Acevedo \\ ORCID: https://orcid.org/0000-0002-1769-2483 \\ Universidade Estadual Paulista, Brasil \\ E-mail: andresgaona80@gmail.com \\ Vander Rocha Lacerda \\ ORCID: https://orcid.org/0000-0003-0109-8030 \\ Universidade Estadual Paulista, Brasil \\ E-mail: vanderroxal@gmail.com \\ Rogerio Lopes Vieites \\ ORCID: https://orcid.org/0000-0002-3865-0426 \\ Universidade Estadual Paulista, Brasil \\ E-mail: rogerio.vieites@unesp.br
}

\begin{abstract}
Resumo
A romã é conhecida por seus efeitos benéficos a saúde, principalmente por suas propriedades antioxidantes e é amplamente cultivada em diferentes regiões do mundo, o que reflete na grande diversidade de composição dos frutos. Desta forma o objetivo do presente trabalho foi avaliar as características físicas, físico-químicas, bioquímicos e composição centesimal da romã cv. Comum cultivada no Oeste Paulista. Os frutos utilizados foram colhidos no estádio de maturação comercial, no município de Alvares Machado, região de Presidente Prudente, estado de São Paulo e avaliados quando ao peso, firmeza, coloração, $\mathrm{pH}$, sólidos solúveis (SS), acidez titulável (AT), Ratio (SS/AT), compostos fenólicos totais, atividade antioxidante por DPPH, antocianina monomérica total, composição mineral, umidade, cinzas, proteínas, lipídeos, fibras, açúcares redutores e valor calórico total. Os frutos de romã cv. Comum apresentaram a casca de coloração laranja e polpa (sarcotesta) vermelho-violeta. O potássio (K) foi o macronutriente predominante na casca e na polpa. A polpa apresentou alto teor de sólidos solúveis e baixo teor de acidez titulável, caracterizando sabor adocicado. A casca apresentou maior teor de compostos fenólicos totais e atividade antioxidante em relação a polpa. A polpa apresentou maior valor calórico total.
\end{abstract}

Palavras-chave: Punica granatum L.; Cv. Comum; Composição nutricional; Compostos fenólicos; Antioxidantes.

\begin{abstract}
Pomegranate is known for its beneficial effects on health, mainly for its antioxidant properties and is widely cultivated in different regions of the world, which reflects the great diversity of fruit composition. Thus, the objective of this work was to evaluate the physical, physicochemical, biochemical and proximate composition of pomegranate cv. Comum cultivated in the west of São Paulo. The fruits used were harvested at the commercial maturation stage, in the municipality of Alvares Machado, Presidente Prudente region, state of São Paulo and evaluated for weight, firmness, color, $\mathrm{pH}$, soluble solids (SS), titratable acidity (TA), Ratio (SS/AT), total phenolic compounds, antioxidant activity by DPPH, total monomeric anthocyanin, mineral composition, moisture, ash, proteins, lipids, fibers, reducing sugars and total caloric value. Pomegranate fruits cv. Comum skin had an orange color and pulp (sarcotesta) red-violet. Potassium (K) was the predominant macronutrient in the skin and pulp. The pulp had a high content of soluble solids and a low content of titratable acidity, characterizing a sweet taste. The peel showed higher content of total phenolic compounds and antioxidant activity in relation to the pulp. The pulp had the highest total caloric value.
\end{abstract}

Keywords: Punica granatum L.; Cv. Comum; Nutritional composition; Phenolic compounds; Antioxidants.

\section{Resumen}

La granada es conocida por sus efectos beneficiosos para la salud, principalmente por sus propiedades antioxidantes y se cultiva ampliamente en diferentes regiones del mundo, lo que refleja la gran diversidad de composición de la fruta. Así, el objetivo de este trabajo fue evaluar la composición física, fisicoquímica, bioquímica y próxima de la granada cv. Comum cultivado en el occidente de São Paulo. Los frutos utilizados fueron cosechados en la etapa de maduración comercial, en el municipio de Alvares Machado, Región Presidente Prudente, estado de São Paulo y evaluados por peso, firmeza, color, pH, sólidos solubles (SS), acidez titulable (TA), Ratio (SS / AT), compuestos fenólicos totales, 
actividad antioxidante por DPPH, antocianina monomérica total, composición mineral, humedad, cenizas, proteínas, lípidos, fibras, azúcares reductores y valor calórico total. Frutos de granada cv. Comum la piel tenía un color anaranjado y pulpa (sarcotesta) rojo-violeta. El potasio (K) fue el macronutriente predominante en la piel y la pulpa. La pulpa tenía un alto contenido de sólidos solubles y un bajo contenido de acidez titulable, caracterizando un sabor dulce. La cáscara mostró mayor contenido de compuestos fenólicos totales y actividad antioxidante en relación a la pulpa. La pulpa tuvo el valor calórico total más alto.

Palabras clave: Punica granatum L.; Cv. Comum; Composición nutricional; Compuestos fenólicos; Antioxidantes.

\section{Introdução}

A romã (Punica granatum L.) é um fruto simbólico desde os tempos antigos (Wu \& Tian, 2017), e segundo Boussaa et al. (2020) ganhou muita atenção como alimento funcional e fonte nutracêutica. É capaz de promover a manutenção de um bom estado de saúde, possui propriedades antioxidantes, anti-inflamatórios, antienvelhecimento, prebióticas, anticancerígenas, fitocompostos que podem prevenir o desenvolvimento de várias doenças crônicas e papel protetor contra distúrbios metabólicos e doenças cardiovasculares (Lavoro et al., 2021).

De acordo com Kalaycioglu e Erim (2017) a principal causa do efeito positivo da romã para a saúde é a sua atividade antioxidante e segundo Lampakis et al. (2021), com a crescente consciência do consumo de alimentos que contenham ingredientes bioativos naturais, como antioxidantes, para melhorar e proteger a saúde humana, frutas como a romã e seus resíduos, são produtos alimentícios essenciais que contêm quantidades substanciais desses bioativos. O consumo in natura e o processamento da romã estão associados as características dos frutos, incluindo aparência física, sabor e compostos nutricionais (Boussaa et al., 2020).

A romã é um fruto não climatérico e deve ser colhido com maturidade adequada (Kumar et al., 2018), já que não amadurece após a colheita (Kahramanoğlu \& Usanmaz, 2016). Está amplamente distribuída ao redor do mundo, portanto apresenta uma ampla diversidade genética, resultando em diferenças na sua composição fitoquímica (Melgarejo-Sánchez et al., 2021), sendo o perfil bioativo influenciado pelo cultivar, região de cultivo, clima, maturidade, práticas de cultivo e condições de armazenamento (Fernandes et al. 2017).

Existem muitos cultivares de romã espalhados pelo mundo (Kandylis \& Kokkinomagoulos, 2020), sendo relatados mais de 500, destacando a "Wonderful" (Pareek et al., 2015). De acordo com Ferreira (2017) no mercado nacional os principais cultivares encontradas são Wonderful, Rubi e Comum. Segundo Suzuki (2016) a romã cv. Comum produz frutos graúdos durante todo o ano, diferente de outros cultivares produzidos fora do Brasil.

Desta forma o objetivo do presente trabalho foi avaliar as características físicas, físico-químicas, bioquímicos e composição centesimal da romã cv. Comum cultivada no Oeste Paulista.

\section{Metodologia}

\subsection{Material vegetal}

Os frutos de romã cv. Comum utilizados neste trabalho, foram colhidos manualmente no estádio de maturação comercial, em pomar comercial no município de Alvares Machado (latitude 22 04' 46" S, longitude 512 28' 19" O e $475 \mathrm{~m}$ de altitude), região de Presidente Prudente, estado de São Paulo, em junho de 2019. Os frutos foram transportados em carro com ar condicionado até o laboratório de Pós-colheita de Frutas e Hortaliças do Departamento de Produção Vegetal da FCA/UNESP de Botucatu, onde foram selecionados aleatoriamente 20 frutos, sem danos físicos e biológicos.

O preparo das amostras para as análises foi realizado pela separação das sementes e da casca, de forma manual, em ambiente sem luz. A polpa (sarcotesta) (Melgarejo et al., 2020) foi extraída pela pressão realizada com auxílio de um rolo de 
massa sobre as sementes. As amostras da casca e polpa de romã foram congeladas em nitrogênio líquido imediatamente após separação e mantidas em freezer com temperatura de $-20^{\circ} \mathrm{C}$ até o momento das análises.

\subsection{Análises físicas, físico-químicas, bioquímicas e centesimal}

O peso dos frutos foi determinado com auxílio de uma balança semi-analítica (marca Owa labor) com carga máxima $2000 \mathrm{~g}$ e divisão de $10 \mathrm{mg}$, com resultados expressos em grama. A firmeza foi determinada com o auxílio do Texturômetro (STEVENS - LFRA Texture Analyser) e os resultados foram expressos em Newton (N). A cor instrumental foi determinada utilizando o colorímetro Konica Minolta (Chroma meter, CR 400/410). Foram considerados três pontos para a casca (região equatorial e nas duas extremidades distantes a $3 \pm 1 \mathrm{~cm}$ da região mediana) em cada fruto ainda inteiro e a polpa (sarcotesta) da romã extraída manualmente pela compressão das sementes. As leituras foram obtidas pelos valores de Luminosidade $\left(\mathrm{L}^{*}\right)$, ângulo de cor Hue e Croma (C*) (Minolta, 1998). As análises foram realizadas em triplicata.

Foi determinado para a polpa da romã, o pH utilizando o potenciômetro digital DMPH - 2, conforme metodologia de Brasil (2008). O teor de sólidos solúveis (SS) com auxílio do refratômetro digital de mesa (Digital Refractometer DR 202), com valores expressos em ${ }^{\circ}$ Brix (AOAC, 2005). A acidez titulável (AT) por titulometria com solução padronizada de $\mathrm{NaOH}$ a $0,1 \mathrm{~N}$, com amostras de $2 \mathrm{~g}$ da polpa homogeneizada, diluída em $100 \mathrm{ml}$ de água deionizada, com resultados expressos em porcentagem, seguindo metodologia do Instituto Adolfo Lutz (2008) e o índice de maturação pela relação entre o teor de sólidos solúveis e acidez titulável (SS/AT). As análises foram realizadas em triplicata.

Foram determinados para polpa e casca da romã cv. Comum o teor de açúcares redutores por metodologia descrita por Nelson (1944) e adaptada por Somogy (1945) e a composição mineral de acordo com a metodologia descrita por Malavolta et al. (1997). As análises foram realizadas em triplicata.

Os extratos utilizados foram preparados com a casca de romã congelada e macerada em nitrogênio e com a polpa de romã congelada em nitrogênio. Para a análise de compostos fenólicos totais (CFT) os extratos foram obtidos utilizando-se 100 $\mathrm{mg}$ de casca e $200 \mathrm{mg}$ de polpa de romã, extraídos com $10 \mathrm{~mL}$ de metanol $40 \%$ e acetona $20 \%$ acidificada com $\mathrm{HCl}$ a $0,1 \mathrm{~N}$, respectivamente. Para a atividade antioxidante (AA) por DPPH os extratos foram obtidos utilizando-se $10 \mathrm{mg}$ de casca e $20 \mathrm{mg}$ de polpa de romã, extraídos com $10 \mathrm{~mL}$ de metanol $20 \%$ e etanol $60 \%$, respectivamente. Os processos de extrações foram feitos em banho ultrassônico por 20 minutos e a mistura foi centrifugada a 6000 rpm durante 15 minutos para CFT e AA, e posteriormente retirou-se o sobrenadante para composição do extrato final. Após prontos os extratos da casca da romã foram diluídos. O processo de extração foi realizado em triplicata.

A determinação de compostos fenólicos totais (CFT) para casca e polpa de romã foi realizada de acordo com o método Folin-Ciocalteu (Singleton et al., 1999) com algumas modificações. Uma alíquota de 0,5 ml dos extratos ou solução padrão de ácido gálico (5 a $100 \mathrm{ug} / \mathrm{ml}$ ) e 2,5 $\mathrm{ml}$ de reagente de Folin-Ciocalteu (diluído em água 1:10) foram adicionadas em tubo de ensaio. Após 5 minutos foram adicionados $2 \mathrm{ml}$ de carbonato de sódio $(4 \%, \mathrm{p} / \mathrm{v})$. Os tubos foram agitados e incubados em sala escura à temperatura ambiente por 2 horas. A absorbância foi medida em espectrofotômetro (BEL SP 1105) a $765 \mathrm{~nm}$ (comprimento de onda pré-definido em varredura). As análises foram realizadas em triplicata. Os resultados foram expressos em mg equivalente de ácido gálico por grama de amostra fresca (mg GAE /g).

A atividade antioxidante (AA) foi determinada para a casca e polpa de romã pelo método DPPH (Brand-Williams et al., 1995). Em tubos de ensaio adicionou-se 3,0 $\mathrm{ml}$ do solvente de extração PA, uma alíquota de 0,5 $\mathrm{ml}$ dos extratos ou solução padrão de Trolox e $0,3 \mathrm{ml}$ do radical DPPH. As misturas foram deixadas durante 45 minutos em temperatura ambiente, em sala escura e a absorbância medida em espectrofotômetro (BEL SP 1105) a $517 \mathrm{~nm}$ contra um branco. Os resultados foram expressos em mg de Trolox por grama de amostra fresca (mg Trolox/g). As análises foram realizadas em triplicata. 
O conteúdo de antocianina monomérica total (AT) foi medido pelo método diferencial de pH (Giusti; Wrolstad, 2001). Uma alíquota de $0,4 \mathrm{~mL}$ de cada amostra de polpa de romã foi levada a pH 1,0 pela adição de $3,6 \mathrm{~mL}$ de solução tampão cloreto de potássio pH 1,0 (0,025 M) e a pH 4,5 por 3,6 mL de solução tampão acetato de sódio pH 4,5 (0,4 M), respectivamente. A absorbância das soluções da mistura de reação foi lida contra água como branco em $510 \mathrm{~nm}$ e a $700 \mathrm{~nm}$, após 15 minutos em repouso, usando um espectrofotômetro (BEL SP 1105) à temperatura ambiente. As análises foram realizadas em triplicata. Os resultados foram expressos como mg equivalente de cianidina-3-glicosídeo por grama de polpa $(\mathrm{mg} \mathrm{Cy} / \mathrm{L})$.

Foi determinado para casca e polpa de romã a umidade e cinzas, seguindo metodologia do Instituto Adolfo Lutz (2008); fibra bruta (AOAC, 1997); lipídeos (AOAC, 1997); proteína bruta (Nogueira \& Souza, 2005) e valor calórico total (Mahan \& Escott-Stump, 2005).

\subsection{Análise estatística}

Os dados obtidos pelas análises de cor, bioquímica e centesimal foram submetidos à análise de variância e a médias comparadas pelo teste $\mathrm{t}(\mathrm{LSD}), \mathrm{p}<0,05$ através do software Sisvar versão 5.6 (Lavras, MG, Brasil).

\section{Resultados e Discussão}

Pode-se observar pela Tabela 1 os valores médios para a romã cv. Comum, referentes ao peso e firmeza dos frutos e a cor da casca e polpa determinados pelos parâmetros luminosidade $\left(\mathrm{L}^{*}\right)$, ângulo de cor Hue e croma, e antocianina monomérica total da polpa.

Tabela 1: Peso e firmeza dos frutos inteiros, coloração da casca e polpa determinada pelos parâmetros Luminosidade, Hue e Croma e antocianina monomérica total (AT) da polpa da romã cv. Comum

\begin{tabular}{|c|c|c|c|c|}
\hline Amostras & & Peso (g) & Firmeza $(\mathbf{N})$ & \\
\hline Fruto & & 358,35 & 8,30 & \\
\hline & Luminosidade & Hue & Croma & $\mathrm{AT}(\mathrm{mg} \mathrm{Cy} / \mathrm{L})$ \\
\hline Casca & $65,99 \mathrm{a}^{*}$ & $74,22 \mathrm{~b}$ & $43,72 \mathrm{a}$ & - \\
\hline Polpa & $35,75 \mathrm{~b}$ & $347,90 \mathrm{a}$ & $12,74 \mathrm{~b}$ & 34,12 \\
\hline
\end{tabular}

*Médias seguidas da mesma letra minúscula na coluna não diferem entre si, pelo teste T (LSD), a 5\% de significância. - não determinado. Fonte: Autores.

A romã cv. Comum apresentou frutos com peso médio de 358,35 gramas e de acordo com Serdar e Usanmaz (2017) o tamanho do fruto está muito relacionado com os cultivares, condições ambientais e condições de cultivo, podendo haver diversos pesos de frutos. Pesos superiores e inferiores aos obtidos neste trabalho foram relatados por Coronado-Reyes et al. (2020) para romã cultivar Wonderful para safras diferentes. Hmid et al. (2018) relataram pesos médios entre 206,6 e 506,67 gramas para 18 cultivares de romã cultivadas no Marrocos. Já Chen et al. (2022) relataram pesos variando de 289,25 a 1.072,35 gramas entre 20 cultivares de romã na China.

Para a firmeza, os frutos apresentaram em média 8,30 N para a romã cv. Comum e de acordo com Nazoori et al. (2020) a firmeza é uma característica importante para as frutas, e está relacionada com o potencial de armazenamento e confere resistência a doenças e danos mecânicos. A firmeza é uma importante propriedade textural da romã, é dependente da variedade, tendo impacto direto na aceitação da fruta pelo consumidor e de acordo com Pareek et al. (2015) um dos principais problemas associados à romã é a perda excessiva de peso, o que pode resultar no endurecimento da casca.

De acordo com Yusef et al. (2020) a luminosidade, mensura cores claras e escuras, o ângulo Hue indica cores puras ou misturadas e croma define a saturação da cor. Para a romã cv. Comum a luminosidade da casca dos frutos apresentaram 
valores maiores que a polpa, o que indica casca mais clara, mais brilhante e polpa mais escura e opaca. $\mathrm{O}$ ângulo de cor hue indicou frutos com casca de coloração laranja e polpa vermelho-violeta. Foi observado valor baixo para o croma da polpa, relacionando-se com a menor intensidade de coloração. Segundo com Holland et al. (2009) não há correlação entre a cor da casca e a cor das sementes, fato este observado neste trabalho. Essas cores podem ser muito diferentes ou semelhantes, dependendo da variedade e de acordo com Kahramanoğlu e Usanmaz (2016), apenas a aparência externa é suficiente para a redução do valor comercial.

O conteúdo de antocianina monomérica total para a polpa da romã cv. Comum apresentou em média 34,12 mg de cianidina-3-glicosídeo por litro de polpa e de acordo com Kandylis e Kokkinomagoulos (2020) a variação na coloração dos diferentes cultivares de romã está relacionada principalmente às diferentes concentrações de antocianina, o que foi constatado por Todaro et al. (2016) com valores variando entre 12,6 e 78,7 mg de cianidina-3-glicosídeo por litro, para acessos diferentes de romã.

A romã é rica em antocianinas que possuem alta capacidade antioxidante (Zhao \& Yuan, 2021), potencialmente benéficas à saúde e responsáveis pela coloração dos produtos obtidos a partir do suco de romã, que influenciam na aceitação sensorial pelos consumidores (Santiago, 2014). Desta forma, segundo Suzuki (2016) o tamanho, a coloração e sanidade dos frutos de romã são aspectos a serem considerados para a compra, sendo requeridos frutos sem manchas, avermelhados e graúdos.

Os teores de macro e micronutrientes da casca e da polpa da romã cv. Comum são diferentes (Tabela 2). Os resultados da composição mineral apontaram que entre os macronutrientes, o potássio $(K)$ e o nitrogênio $(N)$ foram os mais abundantes na casca da romã cv. Comum, e entre os micronutrientes, o manganês (Mn) foi o mineral mais abundante, seguido pelo ferro (Fe) e boro (B).

Tabela 2: Composição mineral da casca e polpa dos frutos de romã cv. Comum

\begin{tabular}{|c|c|c|c|c|c|c|c|c|c|c|c|}
\hline & $\mathbf{N}$ & $\mathbf{P}$ & K & $\mathbf{C a}$ & Mg & $\mathbf{S}$ & $\mathbf{B}$ & $\mathrm{Cu}$ & $\mathrm{Fe}$ & Mn & $\mathbf{Z n}$ \\
\hline AMOSTRAS & & ------ & ----- & $\mathrm{g}^{-1}---$ & ---- & & & -------- & - mg kg & 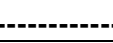 & \\
\hline Casca & 10 & 0,7 & 11 & 2 & 1,2 & 0,8 & 19 & 8 & 30 & 38 & 7 \\
\hline Polpa & 0,533 & 0,116 & 2,674 & 0,019 & 0,053 & 0,054 & - & 0,567 & 0,756 & 0,454 & 0,076 \\
\hline
\end{tabular}

Fonte: Autores.

Para a polpa da romã cv. Comum os resultados da composição mineral apontaram que entre os macronutrientes o potássio $(\mathrm{K})$ e o nitrogênio $(\mathrm{N})$ foram mais abundantes, já para os micronutrientes, o ferro $(\mathrm{Fe})$ foi o mais abundante, seguido do cobre $(\mathrm{Cu})$. Outros autores também relataram o potássio como o elemento predominante em Romãs 'Gabsi' (Boussa et al., 2020), e em vinte cultivares de romã estudadas, cultivadas na Espanha (Alcaraz-Mármol et al., 2017).

Coronado-Reyes et al. (2021) relataram que devido ao alto teor de água e potássio da romã, característica observada também para a romã cv. Comum, e além da baixa concentração de sódio, esta possui propriedades diuréticas e purificantes que, junto com sua concentração em ácido cítrico, favorecem a eliminação de ácido úrico e seus sais através da urina.

Pela Tabela 3 pode-se observar os teores de $\mathrm{pH}$, sólidos solúveis, acidez titulável e índice de maturação (Ratio) para polpa da romã cv. Comum.

Tabela 3: Teores de pH, sólidos solúveis (SS), acidez titulável (AT) e índice de maturação para a polpa dos frutos de romã cv. Comum

\begin{tabular}{lcccc}
\hline & pH & SS $\left({ }^{\circ}\right.$ Brix $)$ & AT $(\%)$ & Ratio \\
\hline Polpa & 2,94 & 12,87 & 0,53 & 24,36 \\
\hline
\end{tabular}

Fonte: Autores. 
A romã cv. Comum apresentou em média pH 2,94, valor intermediário aos encontrados por Fernandes et al. (2017) para Wonderful 2 (2,56) e Valenciana (4,31). O teor de sólidos solúveis para a polpa da romã cv. Comum foi em média 12,87 ${ }^{\circ}$ Brix e a acidez titulável em média $0,53 \%$. De acordo com Smith (2014) o ácido cítrico o mais abundante em quase todos cultivares de romã e segundo Onias et al. (2021) romãs com acidez inferior a 1\% são caracterizadas como doce, exibindo adequada acidez para o consumo in natura. Desta forma a cv. Comum pode ser considerada uma romã doce, Silva et al. (2015) também caracterizaram a romã Molar neste grupo doce.

O índice de maturação para a romã cv. Comum foi em média de 24,36. É um importante parâmetro responsável pelo sabor da romã (Passafiume et al. 2019) e segundo Onias et al. (2021) essa variável expressa o equilíbrio entre a doçura e a acidez da fruta. De acordo Pareek et al. (2015) para a "Wonderful", o índice de maturação deve ser superior a 18,5. Kandylis e Kokkinomagoulos (2020) relataram que a romã apresenta uma acidez relativamente alta (até $20 \mathrm{~g}$ de ácido cítrico/L de suco) e o índice de maturação para a romã, segundo Kahramanoğlu e Usanmaz (2016) é preferível acima de 13, o que foi observado para a romã cv. Comum. De acordo com Mayuoni-Kirshenbaum et al. (2013) as variedades de romã com alta doçura, níveis de acidez moderados a baixos, assim como observadas para a cv. Comum e baixo amargor e adstringência e sementes macias são altamente preferidas.

O teor de compostos fenólicos totais, bem como a atividade antioxidante são superiores para a casca da romã cv. Comum em relação a polpa (Tabela 4).

Tabela 4: Compostos fenólicos totais (CFT) e Atividade antioxidante por DPPH (AA) da casca e polpa dos frutos de romã cv. Comum.

\begin{tabular}{lcc}
\hline & CFT $(\mathbf{m g ~ G A E} / \mathbf{g})$ & AA $(\mathbf{m g}$ Trolox $/ \mathbf{g})$ \\
\hline Casca & $67,13 \mathrm{a}^{*}$ & $32,16 \mathrm{a}$ \\
Polpa & $2,08 \mathrm{~b}$ & $1,27 \mathrm{~b}$ \\
\hline
\end{tabular}

*Médias seguidas da mesma letra minúscula na coluna não diferem entre si, pelo teste T (LSD), a 5\% de significância. Fonte: Autores.

De acordo com Melgarejo-Sánchez et al. (2021) a bioatividade potencial da romã é altamente dependente da variedade e da parte da fruta devido a variabilidade de compostos encontrados em cada variedade e parte da planta, assim como foi observado para os teores da casca e polpa da romã cv. Comum. Segundo Pathak et al. (2017) a casca da romã é fonte de compostos bioativos valiosos, muitos dos quais podem ser convertidos em produtos de valor agregado. A romã apresenta alta concentração de compostos fenólicos com atividade antioxidante (Borguini et al., 2014) e os principais compostos fenólicos da casca da romã incluem flavonóides, taninos e ácidos fenólicos (Singh et al., 2018).

Segundo Suzuki (2016) os consumidores sugerem o fornecimento de informações referentes aos componentes nutracêuticos da romã, seus efeitos e benefícios. E de acordo com Melgarejo-Sánchez et al. (2021) a identificação da capacidade bioativa das diferentes partes da romã é muito importante, pelo fato de que os consumidores possuem distintos hábitos alimentares e para potencializar seu uso em diversos setores, como farmacêutico, alimentício e cosmético.

A composição centesimal da casca e polpa dos frutos para a romã cv. Comum encontram-se na Tabela 5. A polpa apresentou maior teor de umidade em relação a casca. Os teores de cinzas foram superiores para a polpa e a porcentagem de lipídeos foi semelhante para casca e polpa. O teor de proteínas e fibras mostraram-se superiores para a casca da romã. 
Tabela 5: Composição centesimal de Umidade, Cinzas, Lipídeos, Proteína bruta, Fibras, Açúcar redutor e valor calórico total (VCT) da casca e polpa dos frutos de romã cv. Comum

\begin{tabular}{|c|c|c|c|c|c|c|c|}
\hline & Umidade $(\%)$ & Cinzas (\%) & Lipídeos (\%) & $\begin{array}{c}\text { Proteína bruta } \\
(\%)\end{array}$ & Fibras (\%) & $\begin{array}{c}\text { Açúcar } \\
\text { Redutor }(\%) \\
\end{array}$ & VCT (Kcal) \\
\hline Casca & $76,12 b^{*}$ & $1,05 \mathrm{~b}$ & $0,19 \mathrm{a}$ & $2,47 \mathrm{a}$ & $3,32 \mathrm{a}$ & $6,18 \mathrm{~b}$ & $36,32 \mathrm{~b}$ \\
\hline Polpa & $88,80 \mathrm{a}$ & $2,68 \mathrm{a}$ & $0,20 \mathrm{a}$ & $0,79 \mathrm{~b}$ & $0,00 \mathrm{~b}$ & $11,53 \mathrm{a}$ & $51,09 \mathrm{a}$ \\
\hline
\end{tabular}

*Médias seguidas da mesma letra minúscula na coluna não diferem entre si, pelo teste T (LSD), a 5\% de significância. Fonte: Autores.

De acordo com Coronado-Reyes et al. (2021) a romã é um fruto constituído com maiores teores de água e açúcares, sendo menor o teor de lipídios e proteínas, ideal para consumo, com baixo poder calórico, proporções estas também observadas para a romã cv. Comum.

A porcentagem de açúcares e valor calórico são superiores para a polpa em relação a casca para a romã cv. Comum. Segundo Kandylis e Kokkinomagoulos (2020) a glicose e a frutose são os açúcares predominantes nos frutos de romã, o que também foi observado para a cv. Comum.

\section{Conclusão}

De acordo condições do presente trabalho pode-se conclui que: os frutos de romã cv. Comum apresentaram a casca de coloração laranja e polpa (sarcotesta) vermelho-violeta; o potássio (K) foi o macronutriente predominante na casca e na polpa; a polpa apresentou alto teor de sólidos solúveis e baixo teor de acidez titulável, caracterizando sabor adocicado; a casca apresentou maior teor de compostos fenólicos totais e atividade antioxidante em relação a polpa e a polpa apresentou maior valor calórico total.

\section{Agradecimentos}

Ao Conselho Nacional de Desenvolvimento Científico e Tecnológico - CNPq, pelo apoio financeiro para realização do presente trabalho.

\section{Referências}

Alcaraz-Mármol, F., Jáurequi, N. N., García-Sánchez, F., Nicolás, J. J. M. \& Hernadez, F. (2017). Characterization of twenty pomegranate (Punica granatum L.) cultivars grown in Spain: Aptitudes for fresh consumption and processing. Scientia Horticulturae, 219, 152-160. https://doi.org/10.1016/j.scienta.2017.03.008

AOAC International. (1997). Official methods of analysis of AOAC International. Gaithersburg, MD: AOAC International.

AOAC International. (2005). Official methods of analysis of AOAC International. Gaithersburg, MD: AOAC International.

Brand-Wiliams, W., Cuvelier, M.E. \& Berset, C. (1995). Use of a free radical method to evaluate antioxidant activity. Food Science and Technology, 28, 2530 .

Boussaa, F., Zaouay, F., Burlo-Carbonell, F., Noguera-Artiaga, L., Carbonell-Barrachina, A., Melgarejo, P., Hernandez, F. \& Mars, M. (2020). Growing Location Affects Physical Properties, Bioactive Compounds, and Antioxidant Activity of Pomegranate Fruit (Punica granatum L. var. Gabsi). International Journal of Fruit Science, 20, 508-523. https://doi.org/10.1080/15538362.2020.1741058

Chen, Y. H.; Gao, H. F.; Wang, S.; Liu, X. Y.; Hu, Q. X.; Jian, Z. H.; Wan, R.; Song, J. H. \& Shi, J. L. (2022). Comprehensive evaluation of 20 pomegranate (Punica granatum L.) cultivars in China. Journal of Integrative Agriculture, 21 (2), 434-445. https://doi.org/10.1016/S2095-3119(20)63389-5

Coronado-Reyes, J. A., Tinoco-Salazar, J., Guisa-Morales, M. L., Cortés-Penagos, C. J. \& González-Hernández, J. C. (2020). Morphology about the varieties mexican pomegranate fruit (punica granatum) and its ripening stage. Food Science and Technology, p. 1-7. https://doi.org/10.1590/fst.28620

Fernandes, L., Pereira, J. A., Lopéz-Cortés, I., Salazar, D. M., González-Álvarez, J. \& Ramalhosa, E. (2017). Physicochemical composition and antioxidant activity of several pomegranate (Punica granatum L.) cultivars grown in Spain. European Food Research and Technology, 243 , 1799-1814. https://doi.org/10.1007/s00217-017-2884-4

Giusti, M. M. \& Wrolstad, R. E. (2001). Current Protocols in Food Analytical Chemistry, R.E. Wrolstad. 
Hmid, I., Hanine, H., Elothmani, D. \& Oukabli, A. (2018). The physico-chemical characteristics of morrocan pomegranate and evaluation of the antioxidant activity for their juices. Journal of the Saudi Society of Agricultural Sciences, 17 (3), 302-309. https://doi.org/10.1016/j.jssas.2016.06.002

Instituto Adolfo Lutz. (2008). Métodos químicos e físicos para análise de alimentos. (4a ed.). Instituto Adolfo Lutz.

Kahramanoglu, I. \& Usanmaz, S. (2016). Pomegranate production and marketing. CRC Press.

Kalaycioglu, Z. \& Erim, F. B. (2017). Total phenolic contents, antioxidant activities, and bioactive ingredients of juices from pomegranate cultivars worldwide. Food Chemistry, 221, 496-507. https://doi.org/10.1016/j.foodchem.2016.10.084

Kandylis, P. \& Kokkinomagoulos, E. (2020). Food Applications and Potential Health Benefits of Pomegranate and its Derivatives. Foods, 9, 1-21. https://doi.org/10.3390/foods9020122

Lampakis, D., Skenderidis, P. \& Leontopoulos, S. (2021). Technologies and Extraction Methods of Polyphenolic Compounds Derived from Pomegranate (Punica granatum) Peels. A Mini-Review. Processes, 9 (236), 1-14. https://doi.org/10.3390/pr9020236

Lavoro, A., Falzone, L., Gattuso, G., Salemi, R., Cultrera, G., Leone, G. M., Scandurra, G., Candido, S. \& Libra, M. (2021). Pomegranate: A promising avenue against the most common chronic diseases and their associated risk factors (Review). International Journal of Functional Nutrition, 2, 1-12. https://doi.org/10.3892/ijfn.2021.16

Mahan, L. K. \& Escott-Stump S. (2005). Alimentos, nutrição \& dietoterapia. Roca.

Mayuoni-Kirshenbaum, L., Bar-Ya'akov, I., Habit, K., Holland, D. \& Porat, R. (2013). Genetic diversity and sensory preference in pomegranate. Fruits, 68 (6), 517-524. https://doi.org/10.1051/fruits/2013090

Melgarejo, P., Núñez-Gómez, D., Legua, P., Martínez-Nicolás, J. J. \& Almansa, L. (2020). Pomegranate (Punica granatum L.) a dry pericarp fruit with fleshy seeds. Trends in Food Science \& Technology, 102, 232-236. https://doi.org/10.1016/j.tifs.2020.02.014

Melgarejo-Sánchez, P., Núñez-Gómez, D., Martínez-Nicolás, J. J. Hernández, F., Legua, P. \& Melgarejo, P. (2021). Pomegranate variety and pomegranate plant part, relevance from bioactive point of view: a review. Bioresources and Bioprocessing, 8 (2), 1-29. https://doi.org/10.1186/s40643-020-00351-5

Minolta, K. (1998). Comunicação precisa da cor: controle de qualidade da percepção à instrumentação.

Nazoori, F., ZamaniBahramabadi, E., Mirdehghan, S. H. \& Rafe, A. (2020). Extending the shelf life of pomegranate (Punica granatum L.) by GABA coating application. Journal of Food Measurement and Characterization, 14, 2760-2772. https://doi.org/10.1007/s11694-020-00521-1

Nelson, N. (1944). A photometric of the Somogy method for the determination of glucose. Journal of Biological Chemistry, 153, 375-380.

Onias, E. A., Araújo, R. H. C. R., Ferreira, A. P. N., Oliveira, A. M. F., Teodosio, A. E. M. M., Sarmento, D. H. A., Alves, K. A. \& Queiroga, T. B. (2021). Genotype characterization of pomegranate trees grown in Tabuleiro de Russas-CE. Brazilian Journal of Development, 7 (4), $37199-37213$. https://doi.org/10.34117/bjdv7n4-267

Pareek, S., Valero, D. \& Serrano, M. (2015). Postharvest biology and technology of pomegranate. Journal of the Science of Food and Agriculture, 95, 23602379. https://doi.org/10.1002/jsfa.7069

Passafiume, R., Perrone, A., Sortino, G., Saletta, G. G. F., Gentile, C. \& Farina, V. (2019). Chemical-physical characteristics, polyphenolic content and total antioxidant activity of three Italian-grown pomegranate cultivars. NFS Journal, 16, 9-14. https://doi.org/10.1016/j.nfs.2019.06.001

Serdar, H. \& Usanmaz, S. (2017). Effects of Size, Storage Duration, and Modified Atmosphere Packaging on Some Pomological Characteristics of Wonderful Pomegranate Cultivar. Postharvest Handling. https://doi.org/10.5772/67914

Silva, I. M. B. R., Rocha, R. H. C., Silva, H. S., Moreira, I. S., Sousa, F. A. \& Paiva, E. P. (2015). Quality and post-harvest life organic pomegranate 'Molar' produced in Paraiba semiarid. Semina: Ciências Agrárias, 36 (4), 2555-2564. https://doi.org/10.5433/1679-0359.2015v36n4p2555

Singh, B., Singh, J. P., Kaur, A. \& Singh, N. (2018). Phenolic compounds as beneficial phytochemicals in pomegranate (Punica granatum L.) peel: A review. Food Chemistry, 261, 75-86. https://doi.org/10.1016/j.foodchem.2018.04.039

Singleton, V. L., Orthofer, R. \& Lamuela, R. M. (1999). Analysis of total phenol and other oxidation subtrates and antioxidants by means of FolinCiocauteau reagent. Methods of Enzymology, 299, 152-178. https://doi.org/10.1016/S0076-6879(99)99017-1

Somogyi, M. (1945). Determination of blood sugar. Journal Biological Chemistry, 160, 69-73, 1945.

Suzuki, E. T. (2016). Avaliação fenológica, análise econômica e estudo da cadeia produtiva da romã (Punica granatum). (Tese de doutorado). Faculdade de Ciências Agronômicas, Universidade $\quad$ Estadual Paulista, $\quad$ Botucatu, SP, https://repositorio.unesp.br/bitstream/handle/11449/143866/suzuki_et_dr_bot.pdf?sequence=3\&isAllowed=y

Todaro, A., Cavallaro, R., La Malfa, S., Continella, A., Gentile, A., Fischer, U. A., Carle, R. \& Spagna, G. (2016). Anthocyanin profile and antioxidant activity of freshly squeezed pomegranate (punica granatuml.) juices of sicilianand spanish provenancesa. Italian Journal of Food Science, 28 , 464-479. https://doi.org/10.14674/1120-1770/ijfs.v332

Vázquez-Araújo, L., Nuncio-Jáuregui, P. N., Cherdchu, P., Hernández, F., Chambers IV, E. \& Carbonell-Barrachina, A. A. (2014). Physicochemical and descriptive sensory characterization of Spanish pomegranates: aptitudes for processing and fresh consumption. International Journal of Food Science and Technology, 49 (7), 1663-1672. https://doi.org/10.1111/ijfs.12472

Wu, S. \& Tian, L. (2017). Diverse Phytochemicals and Bioactivities in the Ancient Fruit and Modern Functional Food Pomegranate (Punica granatum). Molecules, 22, 1- 17. 2017. https://doi.org/10.3390/molecules22101606

Zhao, X. \& Yuan, Z. (2021). Anthocyanins from pomegranate (Punica granatum 1.) and their role in antioxidant capacities in vitro. Chemistry \& Biodiversity, 18 (10), 1-21. https://doi.org/10.1002/cbdv.202100399 